\title{
Testing differences in relative growth rate: A method avoiding curve fitting and pairing
}

\author{
Hendrik Poorter and Charles Lewis
} Poorter, H. and Lewis, C. 1986. Testing differences in relative growth rate: A method
avoiding curve fitting and pairing. - Physiol. Plant. 67: 223-226.

\begin{abstract}
A method is discussed to test differences in relative growth rates. This method is based on an analysis of variance, with ln-transformed plant weight as dependent variable. A significant Group $\times$ Time interaction indicates differences in relative growth rates between groups. The advantages over the "classical" and "functional" growth analyses are: (1) No pairing procedure is required. (2) More than two groups may be evaluated in one analysis. (3) No decision is required about the polynomial used to fit the data. (4) By partitioning the interaction effect using orthogonal polynomials insight is gained into the nature of differences in relative growth rate. (5) By concentrating attention on the lower order terms of the polynomials, the influence of extraneous variation on conclusions may be minimized.
\end{abstract}

Additional key word - Growth analysis.

H. Poorter, Dept of Plant Physiology, Univ. of Groningen, P.O. Box 14, 9750 AA Haren, The Netherlands; (present address and reprint requests), Dept of Plant Ecology, Univ. of Utrecht, Lange Nieuwstraat 106, 3512 PN Utrecht, The Netherlands; C. Lewis, Dept of Statistics and Measurement Theory (Social Sciences), Univ. of Groningen, Oude Boteringestraat 23, 9712 GC Groningen, The Netherlands.

\section{Introduction}

Two important methods in the analysis of plant growth are the so-called "classical" and "functional" approaches (e.g. Causton and Venus 1981, Hunt 1982). In the functional approach a polynomial of the form $\mathrm{Y}=$ $b_{0}+b_{1} X+b_{2} X^{2}+\ldots+b_{n} X^{n}$ is fitted through the growth data. In this equation the independent variable $\mathrm{X}$ is time, the dependent variable $\mathrm{Y}$ is the $\ln$-transformed weight of the plant. By differentiating this formula an equation for the relative growth rate (RGR) is obtained. One of the problems relating to the functional approach is the choice of the appropriate degree of the polynomial. By selecting a too high order polynomial overfitting is possible. This may result in spurious changes in RGR or net assimilation rates (Nicholls and Calder 1973, Hurd 1977). Another problem, which has received less attention, is related to the statistical test for differences in RGR's. Generally, 95\% confidence bands are computed for the polynomials. However, as a consequence of the computational method used, this will result in a band which is narrowest around the mean of the times and widest at the upper and lower ends of the curve. This characteristic of the bands lacks a biological meaning, and it is difficult to discern when differences in RGR are real or when they are just an artefact of the statistical method.

In the classical approach an RGR is calculated by dividing the difference in In-transformed plant weight at two harvests by the time difference between those harvests. One of the disadvantages of this approach is that, before testing RGR's, deviations have to be calculated. As harvests are destructive, the problem arises that individual replicates of one harvest have to be paired with those of another. This can be done at random, which may result in an overestimation of the variances. Another possibility is to rank them on the basis of their weight and pair plants in sequential weight order in both harvests. However, as differences in weight between plants are not necessarily maintained throughout the

Received 2 October, 1985; revised 30 January, 1986 
growth period (Hunt 1984) the ranking procedure does not seem to be valid. The same objection applies even more strongly to the method of Evans (1972), in which plants are grouped with respect to their size at the beginning of the experiment.

Květ et al. (1971) proposed a method to calculate variances of the RGR, avoiding pairing; this method was later refined by Venus and Causton (1979). A disadvantage is that for adjacent time periods the calculated RGR tends to show large fluctuations, which makes testing for differences rather meaningless. Furthermore, this method only allows testing two RGR's based on weights of just two harvests. Additional information in the form of more treatments or more harvests cannot be evaluated in the same test.

A third method has been used - without further elaboration - by Cain and Ormrod (1984). In the present paper we describe this method in more detail and show that it incorporates the computational advantages of both the classical and the functional approach.

Abbreviations - ANOVA, analysis of variance; RGR, relative growth rate; SS, sum of squares.

\section{Method}

The method is based on an ANOVA carried out with a ln-transformed plant weight or leaf area as dependent variable. In its simplest form this ANOVA contains two factors: Time and (for example) Treatment, both at two levels $\left(a_{1}, a_{2}\right.$ and $b_{1}, b_{2}$, respectively). Then the interaction effect, I, may be defined as

$I=\left(b_{1} a_{2}-b_{1} a_{1}\right)-\left(b_{2} a_{2}-b_{2} a_{1}\right)$

Consider a set of growth data with two treatments and harvests at two times $\left(t_{1}\right.$ and $\left.t_{2}\right)$. If $\ln \mathrm{W}_{\mathrm{ij}}$ represents the mean ln-transformed weight of a set of plants of treatment $\mathrm{i}$ at time $\mathrm{j}$ then equation 1 becomes

$\mathrm{I}=\left(\ln \mathrm{W}_{12}-\ln \mathrm{W}_{11}\right)-\left(\ln \mathrm{W}_{22}-\ln \mathrm{W}_{21}\right)$

This can be rewritten as

$I=\left(t_{2}-t_{1}\right)\left[\frac{\left(\ln W_{12}-\ln W_{11}\right)}{\left(t_{2}-t_{1}\right)}-\frac{\left(\ln W_{22}-\ln W_{21}\right)}{\left(t_{2}-t_{1}\right)}\right]$

thus giving

$\mathrm{I}=\left(\mathrm{t}_{2}-\mathrm{t}_{1}\right)\left[\mathrm{RGR}_{1}-\mathrm{RGR}_{2}\right]$

where $\mathrm{RGR}_{\mathrm{i}}$ indicates the mean RGR of plants of treatment $\mathrm{i}$. Therefore, by testing the sum of squares (SS) due to interaction between Time and Treatment, differences in RGR are tested.

In the case of more than two harvests and/or treatments, the analysis is still applicable. With more than two harvests the $\mathrm{SS}_{\text {interaction }}$ can be examined more closely by performing a trend analysis over time. To that end the data are partitioned using a set of mutually orthogonal contrasts, the so-called "orthogonal polynomials" (Sokal and Rohlf 1969). That is, the interaction effect is described by differences in polynomials $\mathrm{Y}=\mathrm{b}_{0}+\mathrm{b}_{1} \mathrm{X}+$ $b_{2} X^{2}+\ldots+b_{n} X^{n}$. Each term "explains" a part of the $\mathrm{SS}_{\text {interaction. }}$ The linear component indicates a difference in RGR which is maintained throughout the experiment. The quadratic component measures the extent to which differences in RGR change linearly with time. Comparison of the SS of the different terms provides an impression of the nature of the RGR differences.

It is difficult to imagine that the differences in RGR would follow a trend more complicated than could be described by a third order polynomial. Most probably, variance attributed to, e.g., a 4th order term is just an irrelevant deviation. Therefore, fourth and higher order terms generally need not be examined.

\section{An example}

The analysis is illustrated by an experiment described by Poorter and Lambers (1986). The purpose of this experiment was to investigate the influence of different frequencies of changes in nutrient availability on the RGR of two physiologically contrasting inbred lines of Plantago major L. Four treatments were applied. Every eight days six plants were harvested from each treatment and each line. To guard against outlying observations, data were trimmed by excluding the smallest and the largest plant of each group from the analysis (Barnett and Lewis 1978).

Figure 1 shows the time course of the RGR's, calculated in the "classical" way. Table 1 gives the values of the RGR for both lines and results of the $t$-tests performed after calculating the variances according to Venus and Causton (1979) for three periods: day 25-73,

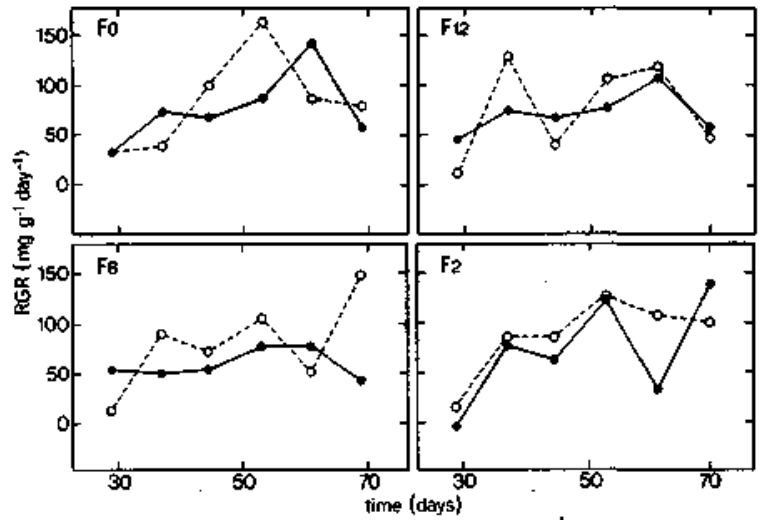

Fig. 1. Time course of the RGR, calculated according to the classical approach. Continuous line, "marginally plastic" genotype; broken line, "highly plastic" genotype. F0, no nutrient level changes; F12, nutrient level change every 12 days; F8, change every eight days; F2, change every 2 days. 
Tab. 1. Values of the relative growth rates $\left(\mathrm{mg} \mathrm{g}^{-1} \mathrm{day}^{-1}\right)$ and their SD of the two inbred lines and four treatments, calculated according to Venus and Causton (1979). MP, "marginally plastic" genotype; HP, "highly plastic" genotype. F0, F12, etc. as in Fig. 1. $\mathrm{P}: P$-value of a $t$-test, testing differences between the two genotypes.

\begin{tabular}{|c|c|c|c|c|c|c|}
\hline \multirow[t]{2}{*}{ Period } & \multirow[t]{2}{*}{ Treatment } & \multicolumn{2}{|c|}{ MP } & \multicolumn{2}{|c|}{ HP } & \multirow[t]{2}{*}{$\mathrm{P}$} \\
\hline & & RGR & SD & RGR & SD & \\
\hline \multirow[t]{4}{*}{ day $25-73$} & F0 & 75.0 & 13.7 & 87.6 & 13.5 & $0.2<P<0.4$ \\
\hline & F12 & 71.5 & 8.2 & 74.4 & 10.2 & $0.5<P<0.9$ \\
\hline & F8 & 57.5 & 8.8 & 77.0 & 5.8 & $P<0.01$ \\
\hline & $\mathrm{F} 2$ & 70.6 & 10.7 & 83.9 & 8.2 & $P<0.05$ \\
\hline \multirow[t]{4}{*}{ day $25-65$} & F0 & 78.5 & 15.1 & 89.5 & 12.2 & $0.2<P<0.4$ \\
\hline & F12 & 74.9 & 8.0 & 80.0 & 10.2 & $0.4<P<0.5$ \\
\hline & F8 & 60.7 & 11.3 & 63.4 & 8.8 & $0.5<P<0.9$ \\
\hline & F2 & 57.1 & 10.4 & 81.1 & 12.6 & $P<0.05$ \\
\hline \multirow[t]{4}{*}{ day $33-73$} & F0 & 84.0 & 16.1 & 89.7 & 15.3 & $0.2<P<0.4$ \\
\hline & F12 & 76.9 & 9.2 & 87.2 & 12.2 & $0.2<P<0.4$ \\
\hline & F8 & 58.6 & 8.8 & 90.7 & 3.7 & $P<0.001$ \\
\hline & F2 & 85.9 & 12.3 & 97.8 & 10.7 & $0.1<P<0.2$ \\
\hline
\end{tabular}

day 25-65 and day 33-73. As these periods overlap almost completely it is expected that the results of the $t$ tests are essentially the same. Especially for treatments F8 and F2 this is not the case. A difference in RGR between the two inbred lines, which is significant if calculated for day 25-73 (Tab. 1, treatment F8) does not approach significance if calculated for day 25-65. Apparently there is too much harvest-to-harvest variation to make this method of testing differences reliable.

Figure 2 shows the time course of the RGR as calculated by the functional approach. Ninety-five \% confidence bands are included. For the computations the computer program of Hunt and Parsons (1974) was used. According to this analysis, none of the treatments introduces significant differences in RGR between the two genotypes.

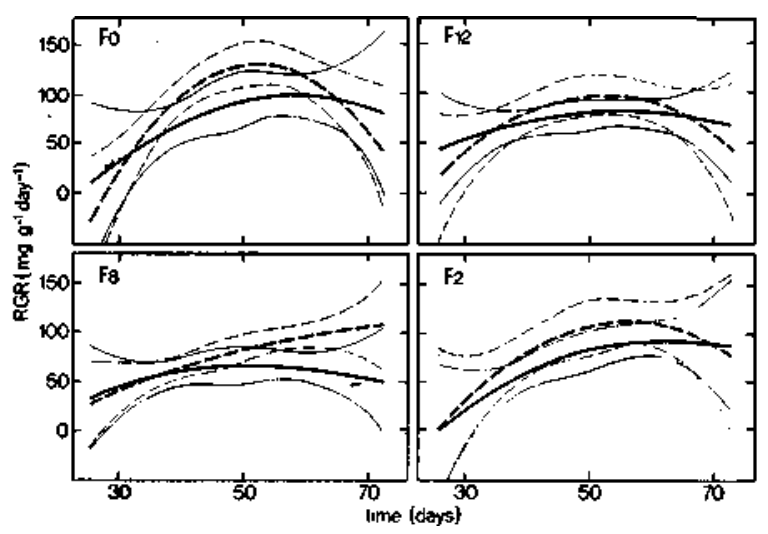

Fig. 2. Time course of the RGR and $95 \%$ confidence bands, calculated according to the functional approach. Continuous line, "marginally plastic" genotype; broken line, "highly plastic" genotype. F0, F12, etc as in Fig. 1.
The ANOVA is given in Tab. 2. In contrast to the results of the functional approach it is concluded from the Genotype $\times$ Time interaction that there is a significant difference in the RGR between the inbred lines. Since most of the $\mathrm{SS}_{\text {interaction }}$ is due to the linear component, it is concluded that this difference in RGR is maintained throughout the whole period examined. The Treatment $\times$ Time interaction is also significant, indicating that the RGR is influenced by the frequency of changes in nutrient availability. The absence of a Genotype $\mathrm{x}$ Treatment $\times$ Time interaction indicates that both inbred lines reacted in the same way to the different treatments.

Tab. 2. ANOVA using data of Poorter and Lambers (1986). Dependent variable: total plant dry weight (after $\ln$ transformation). Independent variables: Genotype, the two inbred lines; Treatment, frequency of nutrient fluctuations; Time, the seven harvests. For the interactions a trend analysis over Time was performed, df, degrees of freedom; $P, P$-value of the $F$ test.

\begin{tabular}{llrrc}
\hline Source of variation & & $\mathrm{SS}$ & $\mathrm{df}$ & $\mathrm{P}$ \\
\hline Genotype & & 0.02 & 1 & 0.646 \\
Treatment & 3.20 & 3 & 0.001 \\
Time & 361.35 & 6 & 0.001 \\
Genotype $\times$ Time: & linear & 2.95 & 1 & 0.001 \\
& quadratic & 0.07 & 1 & 0.420 \\
& residual & 0.27 & 4 & 0.643 \\
Treatment $\times$ Time: & linear & 2.73 & 3 & 0.001 \\
& quadratic & 0.52 & 3 & 0.196 \\
Genotype $\times$ Treatment: & 1.38 & 12 & 0.404 \\
Genotype $\times$ Treatment $\times$ Time: & 1.14 & 3 & 0.018 \\
& linear & 0.30 & 3 & 0.431 \\
& quadratic & 0.24 & 3 & 0.535 \\
Within cells & residual & 1.24 & 12 & 0.500 \\
\hline & & 18.34 & 168 & \\
\hline
\end{tabular}




\section{Conclusions}

The method described above is, with the aid of a computer, a rather straightforward approach to test differences in RGR. It incorporates the advantages of the classical and the functional approach while avoiding curve fitting and pairing and, as can be seen from the example, is more powerful in tracing differences than both forementioned methods.

Acknowledgements - We would like to thank Hans Lambers and Arjen Biere for their critical reading of the manuscript. We thank Cees Hommels for providing the computer program of Hunt and Parsons. Grassland Species Research Group, Publ. No. 101

\section{References}

Barnett, V. \& Lewis, T. 1978. Outliers in Statistical Data. -John Wiley \& Sons, Chichester. p. 48. ISBN 0-471-99599-1.

Cain, N. P. \& Ormrod, D. P. 1984. Hybrid vigour as indicated by early growth characteristics of Populus deltoides, $P$. nigra, and P. x euramericana. - Can. J. Bot. 62: 1-8.

Causton, D. R. \& Venus, J. C. 1981. The Biometry of Plant Growth. - E. Arnold Publishers, London. pp. 16-85. ISBN $0-7131-2844-5$.
Evans, G. C. 1972. The Quantitative Analysis of Plant Growth. - Blackwell Scientific Publications, Oxford. pp. 237-254. ISBN 0-632-06130-8.

Hunt, R. 1982. Plant Growth Curves. The Functional Approach to Plant Growth Analysis. - E. Arnold Publishers, London. pp. 14-120. ISBN 0-7131-2844-5.

- 1984. Relative growth rates of cohorts of ramets cloned from a single genet. - J. Ecol. 72: 299-305.

- \& Parsons, I. T. 1974. A computer program for deriving growth functions in plant growth analysis. - J. Appl. Biol. 11: 297-307.

Hurd, R. G. 1977. Vegetative plant growth analysis in controlled environments. - Ann. Bot. 41: 779-787.

Květ, J., Ondok, J. P., Nečas, J. \& Jarvis, P. G. 1971. Methods of growth analysis. - In Plant Photosynthetic Production; Manual of Methods (Z. Šestak, J. Čatsky and P. G. Jarvis, eds), pp. 343-391. Dr W. J. Junk Publishers, The Hague.

Nicholls, A. O \& Calder, D. M. 1973. Comments on the use of regression analysis for the study of plant growth. - New Phytol. 72: 571-581.

Poorter, H. \& Lambers, H. 1986. Growth and competitive ability of a highly plastic and a marginally plastic genotype of Ptantago major in a fluctuating environment. - Physiol. Plant. 67: 217-222.

Sokal, R. R. \& Rohlf, F. J. 1969. Biometry. - Freeman and Company, San Francisco, pp. 468-475. ISBN 0-71670663-6.

Venus, J. C. \& Causton, D. R. 1979. Plant growth analysis: A reexamination of the methods of calculation of relative growth and net assimilation rates without using fitted functions. - Ann. Bot. 43: 633-638 